\title{
Lower bounds for the number of zeros of cosine polynomials in the period: a problem of Littlewood
}

\author{
by
}

Peter Borwein (Burnaby) and TAmás ErdéLyi (College Station, TX)

1. Introduction. Let $0 \leq n_{1}<n_{2}<\cdots<n_{N}$ be integers. A cosine polynomial of the form $T_{N}(\theta)=\sum_{j=1}^{N} \cos \left(n_{j} \theta\right)$ must have at least one real zero in a period. This is obvious if $n_{1} \neq 0$, since then the integral of the sum on a period is 0 . The above statement is less obvious if $n_{1}=0$, but for sufficiently large $N$ it follows from Littlewood's conjecture simply. Here we mean the conjecture proved by S. Konyagin [5] and independently by McGehee, Pigno, and Smith [11] in 1981. See also [4] for a book proof. It is not difficult to prove the statement in general even in the case $n_{1}=0$. One possible way is to use the identity

$$
\sum_{j=1}^{n_{N}} T_{N}\left((2 j-1) \pi / n_{N}\right)=0 .
$$

See [6], for example. Another way is to use Theorem 2 of [12]. So there is certainly no shortage of possible approaches to prove the starting observation of this paper even in the case $n_{1}=0$.

It seems likely that the number of zeros of the above sums in a period must tend to $\infty$ with $N$. In a private communication B. Conrey asked how fast the number of zeros of the above sums in a period tends to $\infty$ as a function of $N$. In [3] the authors observed that for an odd prime $p$ the Fekete polynomial $f_{p}(z)=\sum_{k=0}^{p-1}\left(\frac{k}{p}\right) z^{k}$ (the coefficients are Legendre symbols) has $\sim \kappa_{0} p$ zeros on the unit circle, where $0.500813>\kappa_{0}>0.500668$. Conrey's question in general does not appear to be easy.

Littlewood in his 1968 monograph "Some Problems in Real and Complex Analysis" [10, problem 22] poses the following research problem, which appears to be still open: "If the $n_{m}$ are integral and all different, what is the lower bound on the number of real zeros of $\sum_{m=1}^{N} \cos \left(n_{m} \theta\right)$ ? Possibly

2000 Mathematics Subject Classification: Primary 41A17.

Key words and phrases: a problem of Littlewood, cosine polynomials, constrained coefficients, number of real zeros. 
$N-1$, or not much less." Here real zeros are counted in a period. In fact no progress appears to have been made on this in the last half-century. In a recent paper [2] we showed that this is false. There exists a cosine polynomial $\sum_{m=1}^{N} \cos \left(n_{m} \theta\right)$ with the $n_{m}$ integral and all different so that the number of its real zeros in the period is $O\left(N^{9 / 10}(\log N)^{1 / 5}\right.$ ) (here the frequencies $n_{m}=n_{m}(N)$ may vary with $\left.N\right)$. However, there are reasons to believe that a cosine polynomial $\sum_{m=1}^{N} \cos \left(n_{m} \theta\right)$ always has many zeros in the period. In this paper we prove the following.

\section{New result}

TheOREm 1. Suppose the set $\left\{a_{j}: j \in \mathbb{N}\right\} \subset \mathbb{R}$ is finite and the set $\left\{j \in \mathbb{N}: a_{j} \neq 0\right\}$ is infinite. Let

$$
T_{n}(t)=\sum_{j=0}^{n} a_{j} \cos (j t) .
$$

Then $\lim _{n \rightarrow \infty} \mathcal{N}\left(T_{n}\right)=\infty$, where $\mathcal{N}(T)$ is the number of zeros of a trigonometric polynomial $T$ in the period $[-\pi, \pi)$.

3. Lemmas and proofs. To prove the new result we need a few lemmas. The first two are straightforward from [4, pp. 285-288] which offers an elegant book proof of the Littlewood conjecture first shown in [5] and [11]. The book [1] deals with a number of related topics. Littlewood [7]-[10] was interested in many closely related problems.

Lemma 3.1. Let $\lambda_{0}<\lambda_{1}<\cdots<\lambda_{m}$ be nonnegative integers and let $S_{m}(t)=\sum_{j=0}^{m} A_{j} \cos \left(\lambda_{j} t\right), \quad A_{j} \in \mathbb{R}, j=0,1, \ldots, m$.

Then

$$
\int_{-\pi}^{\pi}\left|S_{m}(t)\right| d t \geq \frac{1}{60} \sum_{j=0}^{m} \frac{\left|A_{m-j}\right|}{j+1} .
$$

Lemma 3.2. Let $\lambda_{0}<\lambda_{1}<\cdots<\lambda_{m}$ be nonnegative integers and let $S_{m}(t)=\sum_{j=0}^{m} A_{j} \sin \left(\lambda_{j} t\right), \quad A_{j} \in \mathbb{R}, j=0,1, \ldots, m$.

Then

$$
\int_{-\pi}^{\pi}\left|S_{m}(t)\right| d t \geq \frac{1}{60} \sum_{j=0}^{m} \frac{\left|A_{m-j}\right|}{j+1} .
$$

Lemma 3.3. Let $\lambda_{0}<\lambda_{1}<\cdots<\lambda_{m}$ be nonnegative integers and let

$$
S_{m}(t)=\sum_{j=0}^{m} A_{j} \cos \left(\lambda_{j} t\right), \quad A_{j} \in \mathbb{R}, j=0,1, \ldots, m .
$$


Let $A:=\max \left\{\left|A_{j}\right|: j=0,1, \ldots, m\right\}$. Suppose $S_{m}$ has at most $K-1 \geq 0$ zeros in the period $[-\pi, \pi)$. Then

$$
\int_{-\pi}^{\pi}\left|S_{m}(t)\right| d t \leq 2 K A\left(\pi+\sum_{j=1}^{m} \frac{1}{\lambda_{j}}\right) \leq 2 K A(5+\log m) .
$$

Proof. We may assume that $\lambda_{0}=0$; the case $\lambda_{0}>0$ can be handled similarly. Associated with $S_{m}$ in the lemma let

$$
R_{m}(t):=A_{0} t+\sum_{j=0}^{m} \frac{A_{j}}{\lambda_{j}} \sin \left(\lambda_{j} t\right)
$$

Clearly

$$
\max _{t \in[-\pi, \pi]}\left|R_{m}(t)\right| \leq A\left(\pi+\sum_{j=1}^{m} \frac{1}{\lambda_{j}}\right) .
$$

Also, for every $c \in \mathbb{R}$ the function $R_{m}-c$ has at most $K$ zeros in the period $[-\pi, \pi)$, otherwise Rolle's theorem implies that $S_{m}=\left(R_{m}-c\right)^{\prime}$ has at least $K$ zeros in the period $[-\pi, \pi)$. Hence

$$
\begin{aligned}
\int_{-\pi}^{\pi}\left|S_{m}(t)\right| d t & =V_{-\pi}^{\pi}\left(R_{m}\right) \leq 2 K \max _{t \in[-\pi, \pi]}\left|R_{m}(t)\right| \\
& \leq 2 K A\left(\pi+\sum_{j=1}^{m} \frac{1}{\lambda_{j}}\right) \leq 2 K A(5+\log m),
\end{aligned}
$$

and the lemma is proved.

Proof of the theorem when $\left(a_{n}\right)_{n=0}^{\infty}$ is NOT eventually periodic. Suppose the theorem is false. Then there are $k \in \mathbb{N}$, a sequence $\left(n_{\nu}\right)_{\nu=1}^{\infty}$ of positive integers $n_{1}<n_{2}<\cdots$, and even trigonometric polynomials $Q_{n_{\nu}} \in \mathcal{T}_{k}$ with maximum norm 1 on the period such that

$$
T_{n_{\nu}}(t) Q_{n_{\nu}}(t) \geq 0, \quad t \in \mathbb{R} .
$$

We can pick a subsequence of $\left(n_{\nu}\right)_{\nu=1}^{\infty}$ (without loss of generality we may assume that it is the sequence $\left(n_{\nu}\right)_{\nu=1}^{\infty}$ itself) that converges to a $Q \in \mathcal{T}_{k}$ uniformly on the period $[-\pi, \pi)$. That is,

$$
\lim _{\nu \rightarrow \infty} \varepsilon_{\nu}=0 \quad \text { with } \quad \varepsilon_{\nu}:=\max _{t \in[-\pi, \pi]}\left|Q(t)-Q_{n_{\nu}}(t)\right| .
$$

We introduce the formal trigonometric series

$$
\begin{aligned}
& \sum_{j=0}^{\infty} b_{j} \cos \left(\beta_{j} t\right):=\left(\sum_{j=0}^{\infty} a_{j} \cos (j t)\right) Q(t)^{3}, \quad b_{j} \neq 0, j=0,1, \ldots \\
& \sum_{j=0}^{\infty} d_{j} \cos \left(\delta_{j} t\right):=\left(\sum_{j=0}^{\infty} a_{j} \cos (j t)\right) Q(t)^{4}, \quad d_{j} \neq 0, j=0,1, \ldots
\end{aligned}
$$


where $\beta_{0}<\beta_{1}<\cdots$ and $\delta_{0}<\delta_{1}<\cdots$ are nonnegative integers. Since the set $\left\{a_{j}: j \in \mathbb{N}\right\} \subset \mathbb{R}$ is finite, the sets

$$
\left\{b_{j}: j \in \mathbb{N}\right\} \subset \mathbb{R} \text { and }\left\{d_{j}: j \in \mathbb{N}\right\} \subset \mathbb{R}
$$

are finite as well. Hence there are $\varrho, M \in(0, \infty)$ such that

$$
\left|a_{j}\right| \leq M, \quad \varrho \leq\left|b_{j}\right|,\left|d_{j}\right| \leq M, \quad j=0,1, \ldots
$$

Let

$$
K_{\nu}:=\left|\left\{j \in \mathbb{N}: 0 \leq \beta_{j} \leq n_{\nu}\right\}\right|, \quad L_{\nu}:=\left|\left\{j \in \mathbb{N}: 0 \leq \delta_{j} \leq n_{\nu}\right\}\right| .
$$

Since the sequence $\left(a_{n}\right)_{n=0}^{\infty}$ is not eventually periodic, we have

$$
\lim _{\nu \rightarrow \infty} K_{\nu}=\infty \text { and } \lim _{\nu \rightarrow \infty} L_{\nu}=\infty .
$$

We claim that

$$
K_{\nu} \leq c_{1} L_{\nu}
$$

with some $c_{1}>0$ independent of $\nu \in \mathbb{N}$. Indeed, using Parseval's formula and (3.2)-(3.4), we deduce

$$
\begin{aligned}
\frac{1}{\pi} \int_{-\pi}^{\pi} T_{n_{\nu}}(t)^{2} Q(t)^{4} Q_{n_{\nu}}(t)^{2} d t & =\frac{1}{\pi} \int_{-\pi}^{\pi}\left(T_{n_{\nu}}(t) Q(t)^{2} Q_{n_{\nu}}(t)\right)^{2} d t \\
& \geq\left(K_{\nu}-3 k\right) \frac{1}{2} \varrho^{2} \geq \frac{1}{4} \varrho^{2} K_{\nu}
\end{aligned}
$$

for every sufficiently large $\nu \in \mathbb{N}$. Also, (3.1)-(3.4) imply

$$
\begin{aligned}
& \frac{1}{\pi} \int_{-\pi}^{\pi} T_{n_{\nu}}(t)^{2} Q(t)^{4} Q_{n_{\nu}}(t)^{2} d t \\
& \quad=\frac{1}{\pi} \int_{-\pi}^{\pi}\left(T_{n_{\nu}}(t) Q_{n_{\nu}}(t)\right)\left(T_{n_{\nu}}(t) Q(t)^{4}\right) Q_{n_{\nu}}(t) d t \\
& \quad \leq \frac{1}{\pi}\left(\int_{-\pi}^{\pi} T_{n_{\nu}}(t) Q_{n_{\nu}}(t) d t\right)\left(\max _{t \in[-\pi, \pi]}\left|T_{n_{\nu}}(t) Q(t)^{4}\right|\right)\left(\max _{t \in[-\pi, \pi]}\left|Q_{n_{\nu}}(t)\right|\right) \\
& \quad \leq \frac{1}{\pi}\left(\int_{-\pi}^{\pi} T_{n_{\nu}}(t) Q_{n_{\nu}}(t) d t\right)\left(L_{\nu} M+4 k M\right)\left(\max _{t \in[-\pi, \pi]}\left|Q_{n_{\nu}}(t)\right|\right) \leq c_{2} L_{\nu}
\end{aligned}
$$

with a constant $c_{2}>0$ independent of $\nu$ for every sufficiently large $\nu \in \mathbb{N}$. Now (3.5) follows from (3.6) and (3.7).

From Lemma 3.1 we deduce

$$
\int_{-\pi}^{\pi}\left|T_{n_{\nu}}(t) Q(t)^{4}\right| d t \geq c_{3} \varrho \log L_{\nu}-c_{4}
$$


with some constants $c_{3}>0$ and $c_{4}>0$ independent of $\nu \in \mathbb{N}$. On the other hand, using (3.1), Lemma 3.3, (3.2), (3.3), (3.5), and (3.4), we obtain

$$
\begin{aligned}
& \int_{-\pi}^{\pi}\left|T_{n_{\nu}}(t) Q(t)^{4}\right| d t \\
& \leq \int_{-\pi}^{\pi} T_{n_{\nu}}(t) Q_{n_{\nu}}(t)|Q(t)|^{3} d t+\int_{-\pi}^{\pi}\left|T_{n_{\nu}}(t) Q(t)^{3}\right|\left|Q(t)-Q_{n_{\nu}}(t)\right| d t \\
& \leq\left(\int_{-\pi}^{\pi} T_{n_{\nu}}(t) Q_{n_{\nu}}(t) d t\right)\left(\max _{t \in[-\pi, \pi]}|Q(t)|^{3}\right) \\
& \quad+\left(\int_{-\pi}^{\pi}\left|T_{n_{\nu}}(t) Q(t)^{3}\right| d t\right)\left(\max _{t \in[-\pi, \pi]}\left|Q(t)-Q_{n_{\nu}}(t)\right|\right) \\
& \leq c_{5}+c_{6}\left(\log K_{\nu}\right) \varepsilon_{\nu} \leq c_{5}+c_{6}\left(\log \left(c_{1} L_{\nu}\right)\right) \varepsilon_{\nu} \\
& \leq c_{7}+c_{6}\left(\log L_{\nu}\right) \varepsilon_{\nu}=o\left(\log L_{\nu}\right)
\end{aligned}
$$

where $c_{1}, c_{5}, c_{6}$, and $c_{7}$ are constants independent of $\nu \in \mathbb{N}$. Since (3.9) contradicts (3.8), the proof of the theorem is finished in the case when the sequence $\left(a_{n}\right)_{n=0}^{\infty}$ is not eventually periodic.

Proof of the theorem when $\left(a_{n}\right)_{n=0}^{\infty}$ is eventually periodic. The theorem now follows from Lemma 3.4 below.

Lemma 3.4. Let $\left(a_{j}\right)_{j=0}^{\infty}$ be an eventually periodic sequence of real numbers. Suppose the set $\left\{j \in \mathbb{N}: a_{j} \neq 0\right\}$ is infinite. Then, for all sufficiently large $n$, the trigonometric polynomials

$$
T_{n}(t):=\sum_{j=0}^{n} a_{j} \cos (j t)
$$

have at least $c_{8} \log n$ zeros in the period $[-\pi, \pi)$ with a constant $c_{8}>0$ depending only on $\left(a_{j}\right)_{j=0}^{\infty}$.

Proof. It is a well known classical result that for the trigonometric polynomials

$$
Q_{n}(t):=\sum_{j=1}^{n} \frac{\sin (j t)}{j}
$$

we have

$$
\left|Q_{n}(t)\right| \leq 1+\pi, \quad t \in \mathbb{R}, n=1,2, \ldots .
$$

Using the standard way to prove this, it can be easily shown that if $\left(a_{j}\right)_{j=0}^{\infty}$ 
is an eventually periodic sequence of real numbers, then for the functions

$$
S_{n}(t):=a_{0} t+\sum_{j=1}^{n} \frac{a_{j} \sin (j t)}{j}
$$

we have

$$
\left|S_{n}(t)\right| \leq M, \quad t \in[-\pi, \pi), n=1,2, \ldots,
$$

with a constant $M>0$ depending only on $\left(a_{j}\right)_{j=0}^{\infty}$. Observe that $S_{n}^{\prime}(t)=$ $T_{n}(t)$, so Lemma 3.1 (a consequence of the resolution of the Littlewood conjecture) implies that for all sufficiently large $n$,

$$
V_{-\pi}^{\pi}\left(S_{n}\right)=\int_{-\pi}^{\pi}\left|S_{n}^{\prime}(t)\right| d t=\int_{-\pi}^{\pi}\left|T_{n}(t)\right| d t \geq \eta \log n
$$

with a constant $\eta>0$ depending only on $\left(a_{j}\right)_{j=0}^{\infty}$. Combining (3.10) and (3.11) we can easily deduce that there is a $c \in[-M, M]$ such that for all sufficiently large $n$, the function $S_{n}-c$ has at least $(2 M)^{-1} \eta \log n$ distinct zeros in the period $[-\pi, \pi)$. Hence by Rolle's theorem $T_{n}=\left(S_{n}-c\right)^{\prime}$ has at least $(2 M)^{-1} \eta \log n-1$ distinct zeros in the period $[-\pi, \pi)$ for all sufficiently large $n$.

We prove one more result, Theorem 3.6, closely related to Lemma 3.4. In the proof of Theorem 3.6 we need the following observation.

Lemma 3.5. Suppose $k>2 m \geq 0, k$ is even. Let

$$
z_{j}:=\exp \left(\frac{2 \pi j i}{k}\right), \quad j=0,1, \ldots, k-1,
$$

be the $k$ th roots of unity. Suppose $0 \notin\left\{b_{0}, b_{1}, \ldots, b_{k-1}\right\} \subset \mathbb{R}$ and let

$$
Q(z):=z^{m} \sum_{j=0}^{k-1} b_{j} z^{j}
$$

Then there is a value of $j \in\{0,1, \ldots, k-1\}$ for which $\operatorname{Im}\left(Q\left(z_{j}\right)\right) \neq 0$.

Proof. If the statement of the lemma were false, then

$$
z^{m+k-1}(Q(z)-Q(1 / z))=\left(z^{k}-1\right) \sum_{\nu=0}^{2 m+k-2} \alpha_{\nu} z^{\nu} .
$$

Obviously

$$
\begin{aligned}
z^{m+k-1}(Q(z)- & Q(1 / z))=-b_{k-1}-b_{k-2} z-b_{k-3} z^{2}-\cdots-b_{0} z^{k-1} \\
& +b_{0} z^{2 m+k-1}+b_{1} z^{2 m+k}+b_{2} z^{2 m+k+1}+\cdots+b_{k-1} z^{2 m+2 k-2} .
\end{aligned}
$$

Hence

$$
\alpha_{\nu}=-b_{k-1-\nu}, \quad \alpha_{2 m+k-2-\nu}=b_{k-1-\nu}, \quad \nu=0,1, \ldots, k-1 .
$$


Then for $\nu:=m+k / 2-1<k-1$ we have

$$
-b_{k-1-\nu}=b_{k-1-\nu}, \quad \text { that is, } b_{k-1-\nu}=0,
$$

a contradiction.

Theorem 3.6. Let $0 \notin\left\{b_{0}, b_{1}, \ldots, b_{k-1}\right\} \subset \mathbb{R},\left\{a_{0}, a_{1}, \ldots, a_{m-1}\right\} \subset \mathbb{R}$ and

$$
a_{m+l k+j}=b_{j}, \quad l=0,1, \ldots, j=0,1, \ldots, k-1 .
$$

Suppose $k>2 m \geq 0, k$ is even. Let $n=m+l k+u$ with integers $m \geq 0$, $l \geq 0, k \geq 1$, and $0 \leq u \leq k-1$. Then for every sufficiently large $n$,

$$
T_{n}(t):=\operatorname{Im}\left(\sum_{j=0}^{n} a_{j} e^{i j t}\right)
$$

has at least $c_{9} n$ zeros in $[-\pi, \pi)$, where $c_{9}>0$ is independent of $n$.

Proof. Note that

$$
\begin{aligned}
\sum_{j=0}^{n} a_{j} z^{j} & =\sum_{j=0}^{m-1} a_{j} z^{j}+z^{m}\left(\sum_{j=0}^{k-1} b_{j} z^{j}\right) \frac{z^{(l+1) k}-1}{z^{k}-1}+z^{m+l k} \sum_{j=0}^{u} b_{j} z^{j} \\
& =P_{1}(z)+P_{2}(z),
\end{aligned}
$$

where

$$
\begin{aligned}
& P_{1}(z):=\sum_{j=0}^{m-1} a_{j} z^{j}+z^{m+l k} \sum_{j=0}^{u} b_{j} z^{j} \\
& P_{2}(z):=z^{m} \sum_{j=0}^{k-1} b_{j} z^{j} \frac{z^{(l+1) k}-1}{z^{k}-1}=Q(z) \frac{z^{(l+1) k}-1}{z^{k}-1},
\end{aligned}
$$

with

$$
Q(z):=z^{m} \sum_{j=0}^{k-1} b_{j} z^{j}
$$

By Lemma 3.5 there is a $k$ th root of unity $\xi=e^{i \tau}$ such that $\operatorname{Im}(Q(\xi)) \neq 0$. Then for every $K>0$ there is a $\delta \in(0,2 \pi / k)$ such that $\operatorname{Im}\left(P_{2}\left(e^{i t}\right)\right)$ oscillates between $-K$ and $K$ at least $c_{10}(l+1) k \delta$ times, where $c_{10}>0$ is a constant independent of $n$. Now we choose $\delta \in(0,2 \pi / k)$ for

$$
K:=1+\sum_{j=0}^{m-1}\left|a_{j}\right|+\sum_{j=0}^{k-1}\left|b_{j}\right| .
$$

Then

$$
T_{n}(t):=\operatorname{Im}\left(\sum_{j=0}^{n} a_{j} e^{i j t}\right)=\operatorname{Im}\left(P_{1}\left(e^{i t}\right)\right)+\operatorname{Im}\left(P_{2}\left(e^{i t}\right)\right)
$$


has at least one zero on each interval on which $\operatorname{Im}\left(P_{2}\left(e^{i t}\right)\right)$ oscillates between $-K$ and $K$, and hence it has at least $c_{10}(l+1) k \delta>c_{9} n$ zeros on $[-\pi, \pi)$, where $c_{9}>0$ is a constant independent of $n$.

\section{References}

[1] P. Borwein, Computational Excursions in Analysis and Number Theory, Springer, New York, 2002.

[2] P. Borwein, T. Erdélyi, R. Ferguson, and R. Lockhart, On the zeros of cosine polynomials: solution to a problem of Littlewood, Ann. of Math., to appear.

[3] B. Conrey, A. Granville, B. Poonen, and K. Soundararajan, Zeros of Fekete polynomials, Ann. Inst. Fourier (Grenoble) 50 (2000), 865-889.

[4] R. A. DeVore and G. G. Lorentz, Constructive Approximation, Springer, Berlin, 1993.

[5] S. V. Konyagin, On a problem of Littlewood, Izv. Akad. Nauk SSSR Ser. Mat. 45 (1981), 243-265 (in Russian); English transl.: Math. USSR-Izv. 18 (1981), 205-225.

[6] S. V. Konyagin and V. F. Lev, Character sums in complex half-planes, J. Théor. Nombres Bordeaux 16 (2004), 587-606.

[7] J. E. Littlewood, On the mean values of certain trigonometrical polynomials, J. London Math. Soc. 36 (1961), 307-334.

[8] - On the real roots of real trigonometrical polynomials (II), ibid. 39 (1964), $511-552$.

[9] -, On polynomials $\sum^{n} \pm z^{m}$ and $\sum^{n} e^{\alpha_{m} i} z^{m}, z=e^{\theta i}$, ibid. 41 (1966), 367-376.

[10] - Some Problems in Real and Complex Analysis, Heath Math. Monogr., Heath, Lexington, MA, 1968.

[11] O. C. McGehee, L. Pigno, and B. Smith, Hardy's inequality and the $L_{1}$ norm of exponential sums, Ann. of Math. 113 (1981), 613-618.

[12] I. D. Mercer, Unimodular roots of special Littlewood polynomials, Canad. Math. Bull. 49 (2006), 438-447.

Department of Mathematics and Statistics

Department of Mathematics

Simon Fraser University

Burnaby, BC, Canada V5A 1S6

E-mail: pborwein@cecm.sfu.ca

Texas A\&M University College Station, TX 77843, U.S.A.

E-mail: terdelyi@math.tamu.edu

Received on 23.11.2006

and in revised form on 3.3.2007 